\title{
Coronal Spinal Postural Alignment Screening Tool using Markerless Digital Photography
}

Mitsumasa Hida ${ }^{1,2,3,}$, Ayuna Hasegawa ${ }^{2}$, Sachiyo Kamitani ${ }^{2}$, Yumi Kamitani ${ }^{2}$, Kodai Kitagawa ${ }^{1}$, Shogo Okamatsu ${ }^{1}$, Tadasuke Ohnishi ${ }^{4}$, Seigo Minami ${ }^{3}$, Chikamune Wada ${ }^{1}$

${ }^{I}$ Graduate School of Life Science and Systems Engineering, Kyushu Institute of Technology, 2-4 Hibikino, Wakamatsu-ku, Kitakyushushi, Fukuoka, 808-0196, Japan

${ }^{2}$ Takata Kamitani Hospital, 4-16-24 Kamiyamaguchi, Yamaguchicho, Nishinomiya-shi, Hyogo, 651-1421, Japan

${ }^{3}$ Department of Rehabilitation, Osaka Kawasaki Rehabilitation University, 158 Mizuma, Kaizuka-shi, Osaka, 597-0104, Japan

${ }^{4}$ Department of Physical Therapy, Faculty of Social Work Studies, Josai International University, 1 Gumyo, Togane-shi, Chiba 2838555, Japan

\section{A R T I C L E I N F O}

Article history:

Received: 20 December, 2020

Accepted: 04 April, 2021

Online: 15 April, 2021

Keywords:

Adult degenerative scoliosis

Measurement

Photograph

Markerless

\begin{abstract}
A B S T R A C T
Early detection and proper management of adult degenerative scoliosis (ADS) are important for health promotion. This study aims to develop an ADS screening tool from markerless digital photography and verify its reliability. The study included 17 participants. Outer canthus-horizontal angle (OHA) and trapezius-horizontal angle (THA) were calculated from the image of the upper body of the subject in a coronal plane using ImageJ. The Cobb angle was measured to investigate the correlation between OHA and THA. The intraclass correlation coefficient was analyzed to verify the reliability using the values of skilled and unskilled physiotherapists. The study results demonstrated an excellent correlation between THA and Cobb angle. THA also had an almost perfect intra- and interrater reliability. Because scoliosis is characterized by shoulder imbalance and THA is an index that reflects shoulder imbalance, the correlation with Cobb angle was excellent. THA is a scoliosis screening tool that can be used not only in hospitals but also in various places because even unskilled physiotherapists can measure highly reliable values.
\end{abstract}

\section{Introduction}

Human posture changes with age, illness, and disability. Postural changes are accompanied by joint deformities. Knee osteoarthritis and lumbar spondylosis rapidly increase after 40 years of age, causing pain and falls, leading to disability and the need for support for activities related to daily living [1, 2]. Abnormal alignment of the spinal column is classified into hyperkyphosis, which is an abnormality of the sagittal plane, and scoliosis, which is an abnormality of the coronal plane. Hyperkyphosis is common in the elderly, with a prevalence of $20 \%-40 \%$ [3-4], and it has a negative effect on gait, activities of daily living, and respiratory function [5-8]. Adult degenerative scoliosis (ADS) is another common posture abnormality of the

\footnotetext{
*Corresponding Author: Mitsumasa Hida, Graduate School of Life Science and Systems Engineering, Kyushu Institute of Technology, 2-4 Hibikino, Wakamatsuku, Kitakyushu-shi, Fukuoka, 808-0196, Japan. TEL: +81-72-446-6700 FAX: +81-72-446-6767. hidam@kawasakigakuen.ac.jp
}

coronal plane in the elderly, with a reported prevalence of $20 \%$ or more [9]. It has been reported that ADS cause a difference in the cross-sectional area of the left and right erector spinae muscles, leads to fear and anxiety about walking, and has a negative effect on health-related quality of life [10-12].

Because the degree of spinal misalignment increases with age, early detection and preventative approaches are important $[13,14]$. We believe that low-cost early detection and preventative approaches are needed to curb the enormous increase in costs required for medical and long-term care, especially in Japan. Measuring postural alignment using a digital photograph is useful for screening purposes because it does not require a visit to a medical institution, has no side effects, and is inexpensive. However, available ADS screening tools are few. Previous studies have used photographs and iPhones to screen for the degree of idiopathic scoliosis (IS), which requires the attachment of markers to bone indicators or undressing $[15,16]$. In addition, although 
these methods are inexpensive, they require specialists to obtain measurements; thus, performing repeated measurements at a location different from that of medical institutions is difficult. We have previously published a work regarding the development of a measurement method for the resolution of the abovementioned problems. In other words, we are developing a measurement method that does not require markers, participants to undress, and specialists for measurements. This method has the potential to become a tool that allows older people to measure their postural alignment at home in the future. Posture alignment screening tool using markerless digital photography was devised for the measurement of hyperkyphosis and reportedly has excellent reliability [17]. However, it has not yet been applied to the measurement of ADS.

Therefore, we have developed a markerless measurement method that does not require undressing for ADS screening using digital photographs. The purpose of this study is to verify the correlation and reliability of the ADS screening tool that has been developed using X-rays.

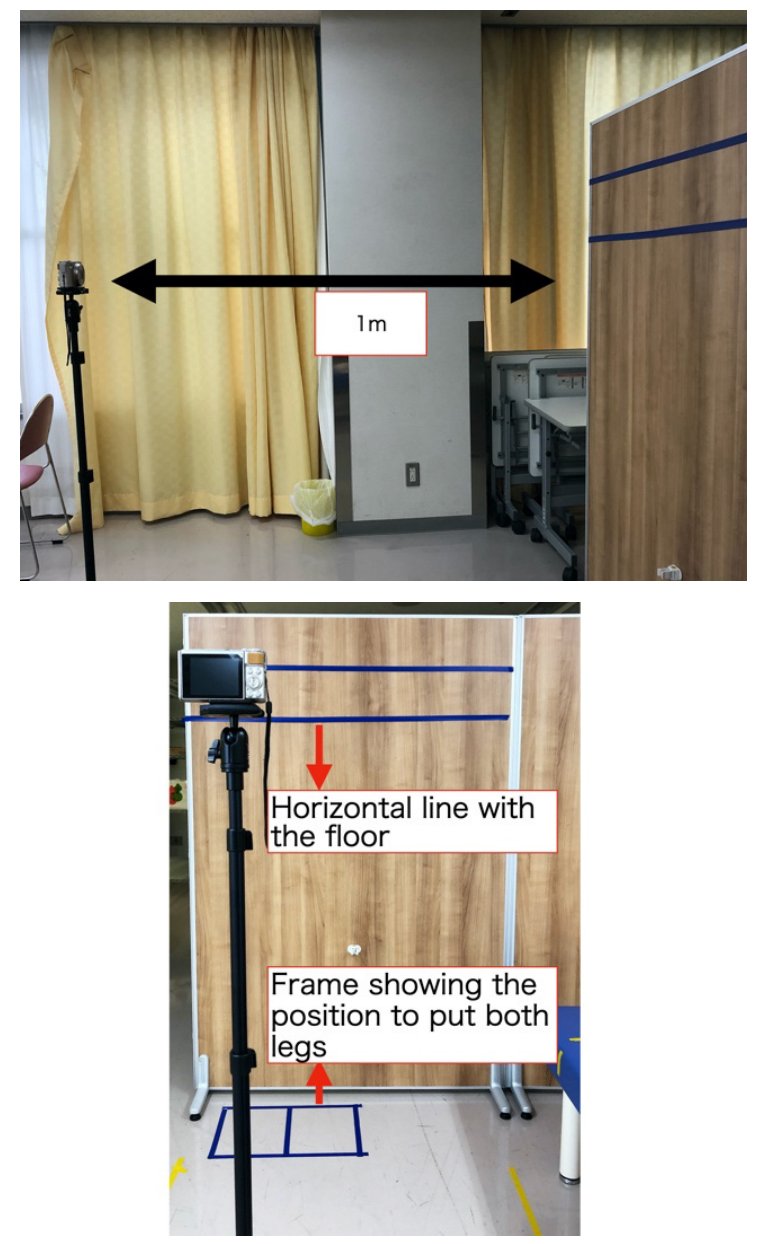

Figure 1: Filming Location

\section{Methods}

\subsection{Subjects}

Subjects were 18 elderly people (5 men and 13 women; mean age, $78.1 \pm 7.4$ years) who visited the outpatient rehabilitation facility. Exclusion criteria included patients with difficulty maintaining a standing position and those with severe dementia.
The examiners were two physiotherapists working at the hospital; examiner A had 2 years of experience as a physiotherapist, and examiner B had 18 years of experience. All participants provided informed consent, and the study was approved by our institutional ethics review board (approval number: OKRU19-A012). This study conforms to the principles of the Declaration of Helsinki.

\subsection{Measurements}

\subsubsection{Coronal Spinal Postural Alignment Measurement Using Digital Photography}

The subjects were asked to be in a standing position. We instructed the subjects to "do not extend the trunk or raise the shoulders" and "keep an eye on the camera lens." They were also instructed to wear a shirt without a collar on the day of the measurement. The digital camera (PowerShot SX740 HS, Canon Inc., Tokyo, Japan) was installed at a position $1 \mathrm{~m}$ away from the subjects (Figure 1).

The line parallel to the floor was prepared in advance and affixed to the wall behind the subject. The resolution of the camera was $1824 \times 1824$ pixels. The focus of the lens was on the subject's eyebrows (Figure 2 ).

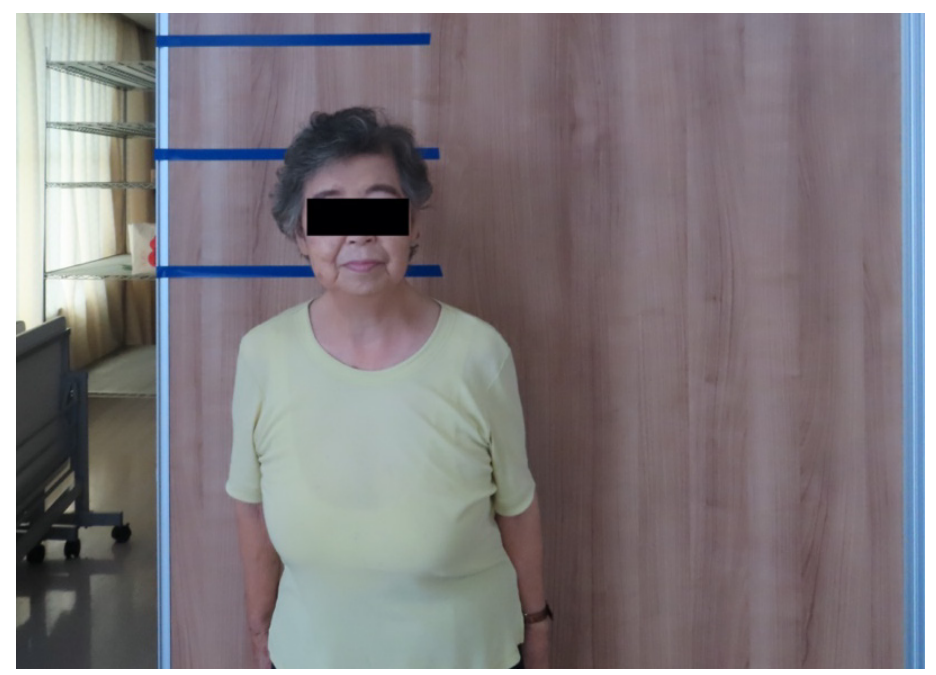

Figure 2: Standing Image Taken to Screen for ADS. The rear line is the horizontal line with the floor.

The images were captured on a PC, and two angles were calculated for ADS screening using ImageJ (version 1.52, National Institutes of Health, Bethesda, MD, USA). According to previous studies, ImageJ has been used for posture assessment, wheelchair sitting posture, and brow position measurements [18-20]. Landmarks for ADS screening were placed on both the lateral canthus and trapezius muscles using the ImageJ point tool. The "find edges" tool was used to accurately point to the landmark (Figure 3). The outer canthus is a landmark used for postural measurement as an index to reflect head position [21]. Head position is related to scoliosis [22]. Because the left and right outer canthus position can change, we used the outer canthus as a landmark tool for screening ADS. In addition, patients with scoliosis are characterized by shoulder imbalance. Because the trapezius angle is one of the key determinants of shoulder imbalance, we chose the trapezius muscle, which reflects the inclination of the shoulder, as a landmark [23]. 
The angle formed by the horizontal line and the line connecting the left and right external canthus angles was called the outer canthus-horizontal angle (OHA). The angle between the trapezius muscle and the horizon for measuring shoulder tilt was called the trapezius-horizontal angle (THA). The right THA was subtracted from the left THA, and the absolute value was calculated (Figure $3 \mathrm{a}, 3 \mathrm{~b})$. A manual was prepared for OHA and THA measurement, and the measurer confirmed it in advance. This manual included an explanation with illustrations for accurately marking the external canthus and trapezius muscles. In addition, ImageJ's operation manual was also published in the manual (Appendix).
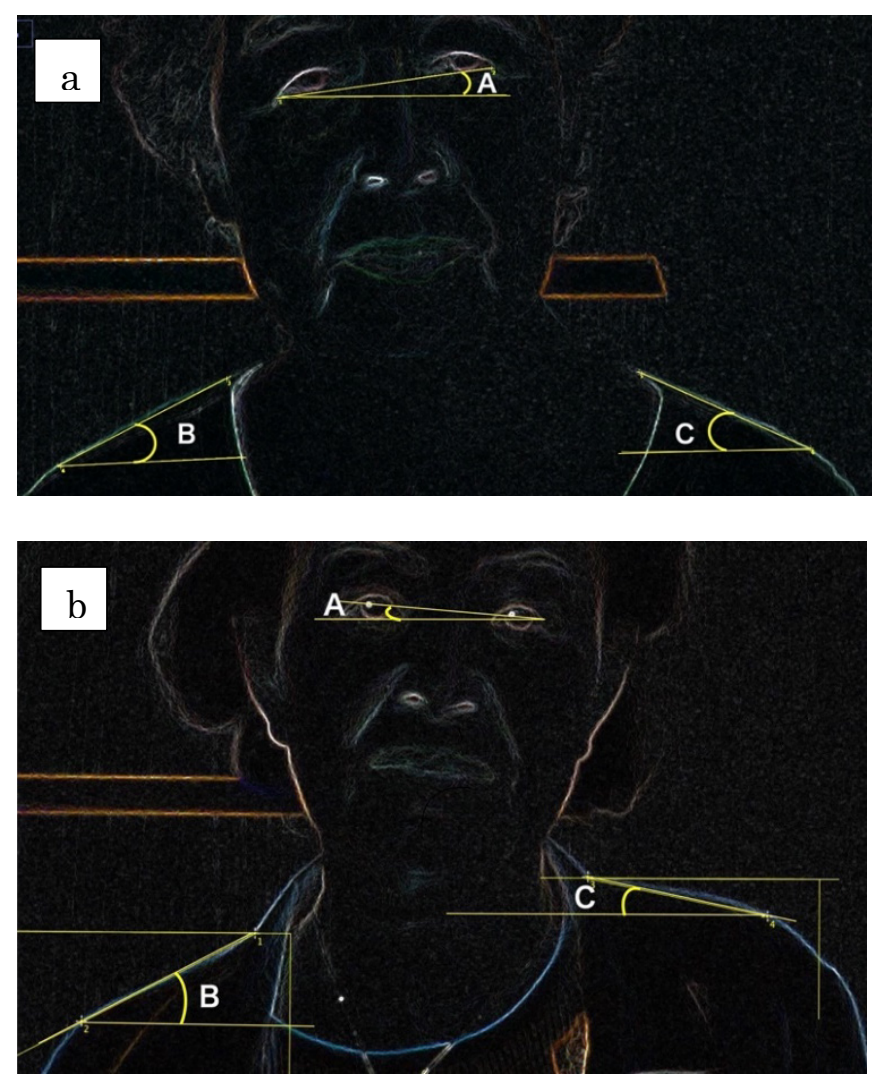

Figure 3: Measurement Using ImageJ's "Find Edges" Tool. A: Outer canthushorizontal angle. B: Trapezius-horizontal angle (right). C: Trapezius-horizontal angle (left). a: normal subject. b: scoliosis subject

\subsubsection{Radiograph}

The Cobb angle was measured from a radiograph taken from the subject's posterior. In American Scoliosis Study Group,

The measurement method was carried out according to the method of the American Scoliosis Study Group [24]. Intersecting perpendiculars were drawn from the superior surface of the proximal end vertebra and the inferior surface of the distal end vertebra of the curve. The angle formed by the perpendiculars was then described as the angle of the scoliotic curve.

\subsubsection{Statistical Analysis}

The relationship between OHA, THA, and Cobb angle was investigated using the Pearson product-moment correlation coefficient. We then calculated the standard error and 95\% confidence interval for the significantly correlated indexes of OHA and THA. The intraclass correlation coefficient (ICC) was used to analyze the interrater reliability (ICC1.1) and intrarater reliability (ICC2.1). The agreement was also tested by plotting the BlandAltman plot. SPSS (version 26.0, IBM, New York, NY, USA) was used for statistical analyses, and the significance level was set to $5 \%$.

\section{Results}

In one subject, accurate measurements could not be made because of the standing posture with hyperextension of the trunk and elevation of the scapula; thus, this subject was excluded from the measurements. Therefore, we analyzed the data obtained from 17 subjects. Table 1 shows the results of each measurement. The overall Cobb angle was $17.3^{\circ} \pm 2.9^{\circ}$, OHA was $2.5^{\circ} \pm 1.0^{\circ}$, and absolute value of THA was $6.9 \pm 5.1$.

Table 1: Characteristics of the subjects

\begin{tabular}{|c|c|}
\hline Age $(\mathrm{y})$ & $78.1 \pm 7.4$ \\
\hline Cobb angle $\left(^{\circ}\right)$ & $17.3 \pm 2.9$ \\
\hline OHA $\left(^{\circ}\right)$ & $2.5 \pm 1.0$ \\
\hline THA of absolute value & $6.9 \pm 5.1$ \\
\hline
\end{tabular}

Mean \pm SD. OHA: outer canthus-horizontal angle, THA: trapezius-horizontal angle

The correlation coefficient between the Cobb angle and OHA was 0.23 , the correlation coefficient for THA was 0.74 , and THA was significantly correlated with the Cobb angle (Table 2). Therefore, the reliability verification was performed only by THA.

Table 2: The relationship with the Cobb angle

\begin{tabular}{|l|l|l|}
\hline & OHA & THA \\
\hline Cobb angle & 0.23 & $0.74^{*}$ \\
\hline \multicolumn{2}{|l|}{ OHA: outer canthus-horizontal angle, THA: trapezius-horizontal angle. ${ }^{*}: \mathrm{p}<0.05$}
\end{tabular}

The interrater reliability (ICC1.1) of THA was 0.98 , and the intrarater reliability (ICC2.1) of THA was 0.90 (Table 3). BlandAltman analyses showed a $100 \%$ agreement, suggesting a perfect correlation between the first and second measurements (Figure 4A) and $94.1 \%$ agreement, suggesting a good correlation between the two examiners (Figure 4B).

Table 3: Intraclass correlation coefficient of THA

\begin{tabular}{|c|c|c|c|c|}
\hline & \multicolumn{2}{|c|}{$\begin{array}{l}\text { Interrater Correlation } \\
\text { Coefficient (ICC } 1,1)\end{array}$} & \multicolumn{2}{|c|}{$\begin{array}{l}\text { Interrater Correlation } \\
\text { Coefficient (ICC2,1) }\end{array}$} \\
\hline & First & Second & $\begin{array}{c}\text { Examiner } \\
\text { A }\end{array}$ & $\begin{array}{c}\text { Examiner } \\
\text { B }\end{array}$ \\
\hline $\begin{array}{l}\text { Mean } \pm \\
\text { SD }\end{array}$ & $\begin{array}{ll}6.9 & \pm \\
5.1 & \end{array}$ & $\begin{array}{cc}6.9 & \pm \\
4.8 & \end{array}$ & $\begin{array}{l}6.9 \\
5.1\end{array}$ & $\begin{array}{l}7.0 \\
4.4\end{array}$ \\
\hline ICC & \multicolumn{2}{|c|}{$0.98 *$} & \multicolumn{2}{|c|}{$0.90^{*}$} \\
\hline $95 \% \mathrm{CI}$ & \multicolumn{2}{|c|}{$0.96-0.99$} & \multicolumn{2}{|c|}{$0.76-0.96$} \\
\hline
\end{tabular}

*: $\mathrm{p}<0.05$. CI: confidence interval

\section{Discussion}

The results of this study indicate that the absolute value of the THA for estimating scoliosis with the shoulder line highly 
correlated with the Cobb angle. Previous studies have noted that scoliosis and shoulder line are related. IS patients had a large angle of shoulder inclination [25]. One of the goals of the surgical treatment of IS is the generation of horizontal shoulders [26].

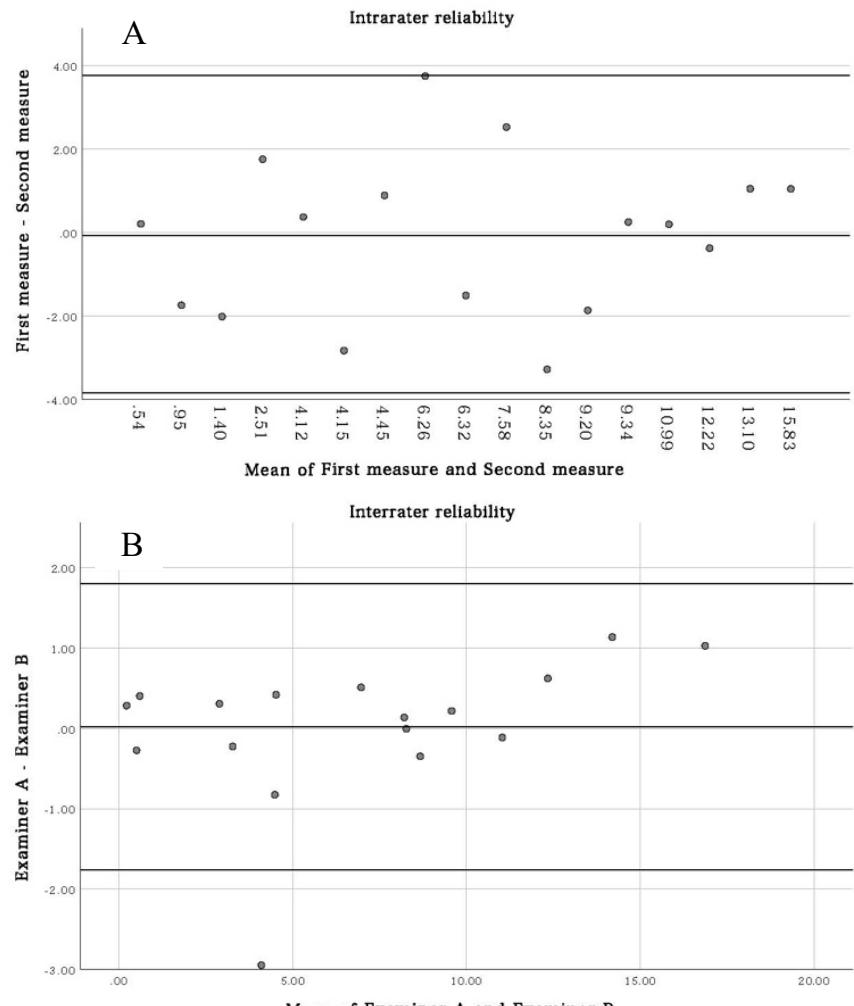

Mean of Examiner A and Examiner B

Figure 4: Bland-Altman plot comparing the mean of two measures (X-axis) to the difference between the two measures (Y-axis). The thick center line represents the mean of the difference between the two measures, and the thick top and bottom lines represent the limits of agreement (mean $\pm 2 \mathrm{SD}$ of difference). There was perfect agreement between the two measures and between the first and second measures.

However, thus far, to the best of our knowledge, there has been no tool developed for screening ADS using the shoulder line. Considering the shoulder alignment mismatch in patients with IS reported in previous studies, we attempted to estimate scoliosis by selecting the shoulder inclination angle as a landmark. Defining the start and end points of the markerless shoulder slope was a challenge, but the manual for improving measurement discrepancies and ImageJ's "find edges" tool contributed to the excellent intra- and interrater reliability, while the Bland-Altman analysis showed perfect agreement. ImageJ's "find edges" tool can utilize the Sobel edge detector to emphasize sudden changes in the brightness of an image [27]. The "find edges" tool is one of the tools used to quantify images in the field of cell biology, but it may also be applied to postural analysis [28-29]. The images taken in this study included the participant and the wall, and it was concluded that the varying brightness between the shoulder line and the wall contributed to the accurate measurement. The intrarater and interrater reliability of THA were almost perfect in the Landis classification [30]. Our proposed ADS screening tool has the advantage of being markerless and not requiring undressing. In addition, the examiners in this study included one experienced physiotherapist and one inexperienced physiotherapist. THA will provide reliable measurements even for examiners with inadequate clinical skills.
On the other hand, we found that the external canthus used to screen for scoliosis from the subject's head position is an inappropriate landmark. We believe that this is because of the righting reflex. The human head tends to maintain a median position in relation to the position of the trunk and neck via the righting reflex. Because the righting reflex involuntarily changes the subject's head position, any facial area is considered unsuitable for screening for scoliosis.

Using digital images for measurement purposes is inexpensive, and when performed according to procedure, measurements can be made by a nonexpert and can be repeatedly made, unlike X-rays, which expose subjects to radiation. In other words, posture alignment measurement using digital images may be possible at various locations outside a medical institution and perhaps even at home. This measurement method may be effectively aligned with the attitudes of many countries that are taking measures against COVID-19 spread. In addition, the repeated measurable advantage is that, by confirming the regular posture alignment, increasing the subject's motivation to improve their posture may become possible. To measure postural alignment in various locations without the need to visit a medical institution, a markerless method to minimize undressing is required. Because the new scoliosis screening tool developed in this study meets these conditions, it has the advantage of reducing medical costs for measuring postural alignment abnormalities. In addition, this method of measurement may aid in early detection and will decrease the number of patients with ADS in the future.

This study is limited by the fact that the physiotherapist was an examiner. Physical therapists employ techniques to analyze the patient posture in detail [31]. To allow generalization of the results of the present study, different individuals need to serve as examiners, and we are currently conducting research for this generalization. We used the measurement manual for generalization but used ImageJ for angle screening. Although Image J is freeware, it has barriers to its use by nonexperts. Furthermore, the THA proposed in this study cannot be used for accurate diagnosis because it cannot identify the site of the curved spinal column. However, we are convinced that THA is a simpler method for screening ADS and a more compliant measurement for the subject, making it a suitable technique for early detection and regular postural observation. In the future, we will develop an application that measures hyperkyphosis and ADS using the digital camera of a smartphone.

\section{Conclusion}

The present study aims to develop an ADS screening tool from markerless digital photography and verify its reliability. Our results indicate an excellent correlation between THA and Cobb angle. THA is a scoliosis screening tool that can be used not only in hospitals but also in various other venues, because even unskilled physiotherapists can obtain highly reliable measurements.

\section{Conflict of Interest}

The authors declared no potential conflicts of interest with respect to the research, authorship, and/or publication of this article.

\section{Acknowledgment}

This research was carried out by the Osaka Kawasaki Rehabilitation University Joint Research Grant. We appreciate its 
support.

\section{References}

[1] N. Yoshimura et al., "Prevalence of knee osteoarthritis, lumbar spondylosis, and osteoporosis in Japanese men and women: the research on osteoarthritis/osteoporosis against disability study," Journal of Bone and Mineral Metabolism, 27(5), 620-628, 2009, doi:10.1007/s00774-009-00808.

[2] Ministry of Health, Labour and Welfare, The outline of the results of National Livelihood Survey 2019. https://www.mhlw.go.jp/toukei/saikin/hw/k-tyosa/k-tyosa19/index.html, 2019

[3] T. Ailon, C.I. Shaffrey, L.G. Lenke, J.S. Harrop, J.S. Smith, "Progressive spinal kyphosis in the aging population," Neurosurgery, 77(4), S164-S172, 2015, doi:10.1227/NEU.0000000000000944.

[4] Y. Kasukawa et al., "Age-related changes in muscle strength and spinal kyphosis angles in an elderly Japanese population," Clinical Interventions in Aging, 20(12), 413-420, 2017, doi:10.2147/CIA.S113352.

[5] S. Ota et al., "Relationship between standing postural alignments and physical function among elderly women using day service centers in Japan," Journal of Back and Musculoskeletal Rehabilitation, 28(1), 111-117, 2015, doi:10.3233/BMR-140498.

[6] C. McDaniels-Davidson, A. Davis, D. Wing, C. Macera, S.P. Lindsay, J.T. Schousboe, J.F. Nichols, D.M. Kado, "Kyphosis and incident falls among community-dwelling older adults," Osteoporosis International, 29(1), 163169, 2018, doi:10.1007/s00198-017-4253-3.

[7] K. Sugai, T. Michikawa, T. Takebayashi, M. Matsumoto, M. Nakamura, Y. Nishiwaki, "Association between visual classification of kyphosis and future ADL decline in community-dwelling elderly people: the Kurabuchi study," Archives of Osteoporosis, 14(1), 3, 2018, doi:10.1007/s11657-018-0551-4.

[8] A.L. Lorbergs, G.T. O’Connor, Y. Zhou, T.G. Travison, D.P. Kiel, L.A. Cupples, H. Rosen, E.J. Samelson, "Severity of kyphosis and decline in lung function: the Framingham study," Journals of Gerontology Series A: Biomedical Sciences and Medical Sciences, 72(5), 689-694, 2017, doi:10.1093/gerona/glw124.

[9] J. Urrutia, T. Zamora, I. Klaber, "Thoracic scoliosis prevalence in patients 50 years or older and its relationship with age, sex, and thoracic kyphosis," Spine, 39(2), 149-152, 2014, doi:10.1097/brs.0000000000000095.

[10] H. Kim, C.K. Lee, J.S. Yeom, J.H. Lee, J.H. Cho, S.I. Shin, H.J. Lee, B.S. Chang, "Asymmetry of the cross-sectional area of paravertebral and psoas muscle in patients with degenerative scoliosis," European Spine Journal, 22(6), 1332-1338, 2013, doi:10.1007/s00586-013-2740-6.

[11] R. Haddas, I.H. Lieberman, A. Block, "The relationship between fearavoidance and neuromuscular measures of function in patients with adult degenerative scoliosis," Spine 43(23), E1412-E1421, 2018, doi:10.1097/BRS.0000000000002719.

[12] F. Schwab, A. Dubey, M. Pagala, L. Gamez, J.P. Farcy, "Adult scoliosis: a health assessment analysis by SF-36," Spine 28(6), 602-606, 2003, doi:10.1097/01.BRS.0000049924.94414.BB.

[13] G.C. Robin, Y. Span, R. Steinberg, M. Makin, J. Menczel, "Scoliosis in the elderly: a follow-up study," Spine 7(4), 355-359, 1982, doi:10.1097/00007632-198207000-00005.

[14] C. Tüzün, I. Yorulmaz, A. Cindaş, S. Vatan, "Low back pain and posture," Clinical Rheumatology, 18(4), 308-312, 1999, doi:10.1007/s100670050107.

[15] A. Prowse, R. Pope, P. Gerdhem, A. Abbott, "Reliability and validity of inexpensive and easily administered anthropometric clinical evaluation methods of postural asymmetry measurement in adolescent idiopathic scoliosis: a systematic review," European spine journal, 25(2), 450-466, 2016, doi:10.1007/s00586-015-3961-7.

[16] P.H. Heitz, J.F. Aubin-Fournier, É. Parent, C. Fortin, "Test-retest reliability of posture measurements in adolescents with idiopathic scoliosis," Spine Journal, 18(12), 2247-2258, 2018, doi:10.1016/j.spinee.2018.05.006.

[17] M. Hida, S. Kawashima, K. Kitagawa, S. Okamatsu, R. Imai, S. Minami, T. Ohnishi, C. Wada, "Spinal postural alignment measurements using makerless digital photography," Journal of Orthopaedic Surgery (Hong Kong). 28(3), 2309499020960834, 2020

[18] E.B. Garrison, J. Dropkin, R. Russell, P. Jenkins, "Modified path methodology for obtaining interval-scaled postural assessments of farmworkers," Journal of Agricultural Safety and Health, 24(1), 43-52, 2018, doi: $10.13031 /$ jash. 12453 .

[19] N.S. Yoon, H.B. Ahn, "Exploring brow position changes with age in Koreans. Korean Journal of Ophthalmology, 33, 91-94, 2019, doi:10.3341/kjo.2018.0013.
[20] A.M. Alkhateeb, B.J. Forrester, N. Daher, B.D. Martin, A.A. Alonazi, "Validity and reliability of wheelchair sitting posture measures using Coach's Eye in abled subjects," Assistive Technology, 29, 210-221, 2017, doi:10.1080/10400435.2016.1220994.

[21] Z. Hazar, G.O. Karabicak, U. Tiftikci, "Reliability of photographic posture analysis of adolescents," Journal of Physical Therapy Science, 27, 3123 3126, 2015, doi:10.1589/jpts.27.3123.

[22] S. Wang, G. Lin, Y. Yang, S. Cai, Q. Zhuang, Y. Tian, J. Zhang, "Outcomes of $360^{\circ}$ osteotomy in the cervicothoracic spine (c7-t1) for congenital cervicothoracic kyphoscoliosis in children. The Journal of Bone and Joint Surgery7;101(15):1357-1365, 2019, doi: 10.2106/JBJS.18.01428.

[23] K.V. Menon, H.M. Pillay, M. Anbuselvam, N. Tahasildar, "Post-operative shoulder imbalance in adolescent idiopathic scoliosis: a study of clinical photographs," Scoliosis 17, 10, 1-9, 2015,. doi: 10.1186/s13013-015-00556.

[24] P.J. Scholten, A.G. Veldhuizen, "Analysis of Cobb angle measurements in scoliosis," Clinical Biomechanics, 2(1), 7-13, 1987, doi:10.1016/02680033(87)90039-8.

[25] P.J. Penha, N.L.J. Penha, B.K.J. De Carvalho, R.M. Andrade, A.C.B. Schmitt, S.M.A. João, "Posture alignment of adolescent idiopathic scoliosis: photogrammetry in scoliosis school screening," Journal of Manipulative and Physiological Therapeutics, 40(6), 441-451, 2017, doi:10.1016/j.jmpt.2017.03.013.

[26] P.D. Trobisch, A.R. Ducoffe, B.S. Lonner, T.J. Errico, "Choosing fusion levels in adolescent idiopathic scoliosis," Journal of the American Academy of Orthopaedic Surgeons, 21(9), 519-528, 2013, doi:10.5435/JAAOS-21$09-519$.

[27] T. Ferreira, W.S. Rasband, "ImageJ User Guide - IJ 1.46. U.S. National Institutes of Health; 2010-2012. imagej.nih.gov/ij/docs/guide/

[28] E.T. Arena, C.T. Rueden, M.C. Hiner, S. Wang, M. Yuan, K.W. Eliceiri, "Quantitating the cell: turning images into numbers with ImageJ. Wiley Interdisciplinary Reviews-Developmental Biology, ;6(2), 2017, doi: $10.1002 / w d e v .260$.

[29] S.N. Omkar, M.M. Kumar, D. Mudigere, "Postural assessment of arbitrarily taken portrait and profile photographs using ImageJ," Journal of Bodywork and Movement Therapies, 11(3):231-237, 2007

[30] J.R. LandisG.G. Koch, "The measurement of observer agreement for categorical data," Biometrics, 33(1), 159-174, 1977, doi: 10.2307/2529310.

[31] V. KorakakisK. O'Sullivan, P.B. O'Sullivan, V. Evagelinou, Y. Sotiralis, A Sideris, K. Sakellariou, S. Karanasios, G. Giakas, "Physiotherapist perceptions of optimal sitting and standing posture," Musculoskeletal Science and Practice, 39, 24-31, 2019, doi: 10.1016/j.msksp.2018.11.004 
Appendix

Procedure of Scoliosis Measurement

1. Preparation for the measurement of scoliosis

(1) Put a sticker on the wall to show the horizon.

(2) Create an index for standing with both legs shoulderwidth apart.

(3) Place the digital camera $1 \mathrm{~m}$ away from the wall.

2. Taking a photograph

(Please be prepared to wear light clothes on the day of shooting [e.g., a T-shirt]).

(1) Focus on the subject's eyebrows.

(2) Instruct them to stand in their usual posture.

(3) Instruct them to keep their eyes open.

3. Trapezius-Horizontal Angle (THA) Measurement tool (Please install ImageJ on your PC in advance)

(1) Draw a horizontal line.

(2) Make points at both ends of the shoulder line, which represents the trapezius muscle.

(3) Measure THA.

(4) Calculate the absolute value of the difference between the left and right THAs.

* Ensure that the shoulder line does not mix with the neck and upper arm lines.

(Creation Date: 9/10/2020)

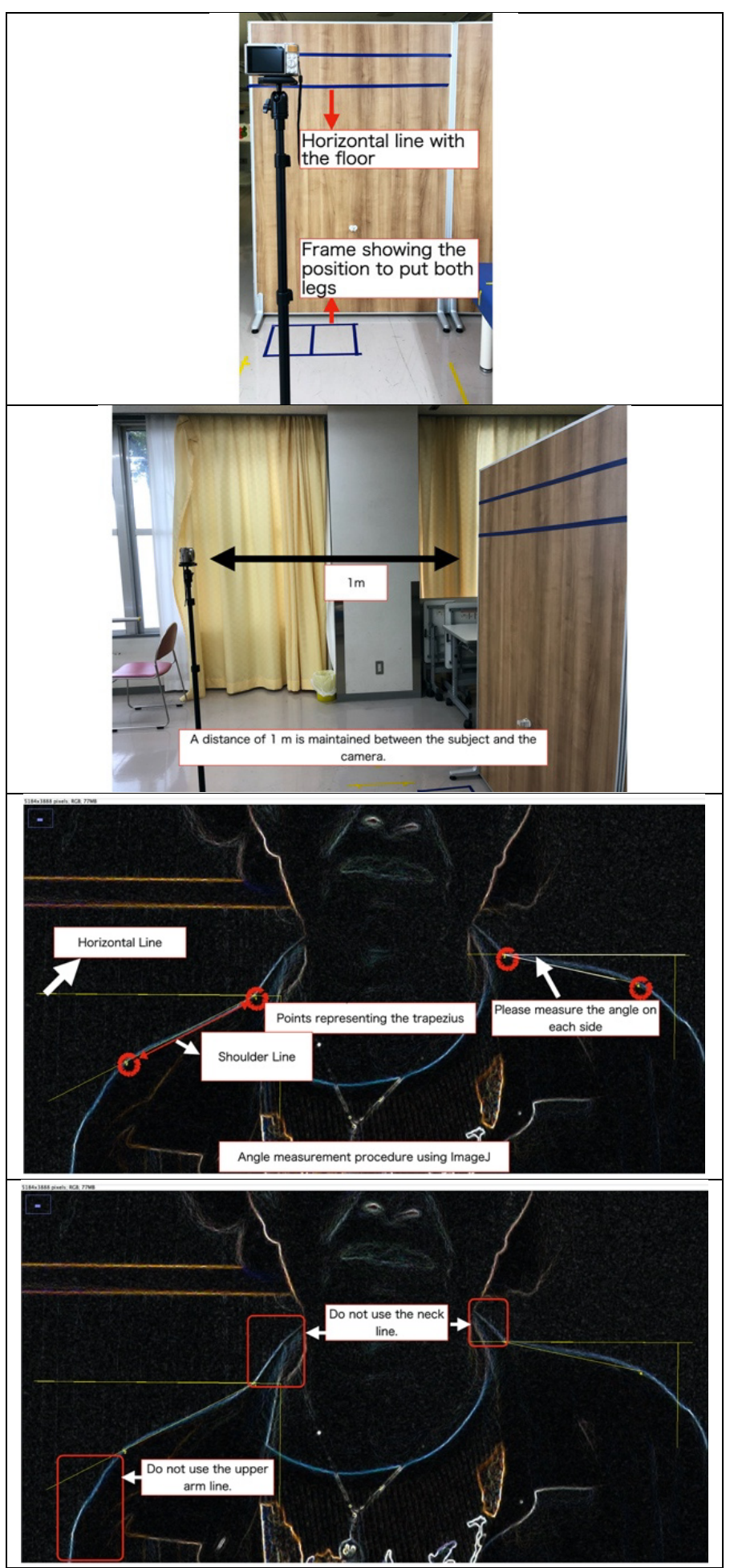

